\title{
Large volume spacer devices and the influence of high dose beclomethasone dipropionate on hypothalamo-pituitary-adrenal axis function
}

\author{
Peter H Brown, Andrew P Greening, Graham K Crompton
}

\begin{abstract}
Background The systemic effects of the inhaled corticosteroid beclomethasone dipropionate are reduced if the drug is inhaled through a large volume spacer. Use of spacers may therefore permit higher doses to be given without causing hypothalamo-pituitary-adrenal suppression.
\end{abstract}

Methods Randomised, double blind, double dummy, parallel group study was carried out in adults with chronic asthma to determine the dose of beclomethasone dipropionate causing hypothalamopituitary-adrenal suppression when the drug was administered by metered dose aerosol with and without a large volume spacer. After a four week run in taking $1.5 \mathrm{mg}$ beclomethasone daily 50 patients underwent tests of hypothalamo-pituitary-adrenal function (measurement of $0900 \mathrm{~h}$ serum cortisol concentration and 24 hour urinary free cortisol excretion and the short tetracosactrin test). Six patients had hypothalamo-pituitaryadrenal suppression (results of at least two tests subnormal) and asthma was well controlled in six others. Thirty eight patients received increasing doses of beclomethasone with (group S) or without (group MDI) a $750 \mathrm{ml}$ spacer. The daily dose was increased by $0.5 \mathrm{mg}$ at monthly intervals until hypothalamopituitary-adrenal suppression developed or a dose of $5 \mathrm{mg} /$ day was achieved. Asthma symptoms and peak expiratory flow were recorded daily.

Results Twenty three patients completed the study, one (group S) reaching a dose of $5 \mathrm{mg}$ beclomethasone dipropionate daily without hypothalamopituitary-adrenal suppression. Reasons for withdrawal were poor compliance $(n=10)$, the patient's decision $(n=3)$, and asthma that was too unstable $(n=2)$. "Intention to treat" analysis showed that the median dose of beclomethasone causing hypothalamo-pituitary-adrenal suppression was similar in the two groups $(3.25 \mathrm{mg}$ in $\mathrm{S} v 3.0 \mathrm{mg}$ in $\mathrm{MDI}$; $95 \%$ confidence interval (CI) for difference -1.0 to $1.0 \mathrm{mg}$ ). At $2 \mathrm{mg} /$ day of beclomethasone most patients in both groups had well controlled asthma and there were no differences in symptoms or peak flow between the groups. Good control at this dose did not permit conclusions to be drawn about the efficacy of higher doses.

Conclusions There is wide interindividual variation in the dose of beclomethasone dipropionate causing hypothalamopituitary-adrenal suppression. Whether or not a spacer is used, doses higher than the currently accepted maximum of 2 mg/day can be taken by many adults with asthma without causing subnormal function of the hypothalamo-pituitaryadrenal axis. Whether these higher doses are more effective in controlling asthma remains to be established.

(Thorax 1993;48:233-238)

High dose inhaled corticosteroids (more than $1 \mathrm{mg}$ beclomethasone dipropionate or budesonide daily) are being used more frequently in the treatment of chronic asthma. Early studies $^{12}$ suggested that most patients taking $2 \mathrm{mg}$ beclomethasone had impaired function of the hypothalamo-pituitary-adrenal axis and thus $2 \mathrm{mg} /$ day became the maximum recommended dose. In a recent survey, however, of adults with asthma taking long term high dose inhaled corticosteroids ${ }^{3}$ most of those inhaling $2 \mathrm{mg}$ beclomethasone daily were found to have normal hypothalamo-pituitaryadrenal function. In that study only a few patients were using large volume spacers and there is now evidence that these devices can protect against the adverse effects of beclomethasone on the hypothalamo-pituitary-adrenal axis. ${ }^{4-7}$ Our study has attempted to answer three questions: (1) What daily dose of beclomethasone dipropionate will cause subnormal hypothalamo-pituitaryadrenal function in asthmatic adults having long term treatment? (2) Can the protective effect of a spacer shown in open studies of asthmatic patients ${ }^{457}$ be confirmed in a randomised, double blind study? (3) Is administration of high dose inhaled corticosteroid via a spacer clinically more effective than conventional treatment with a metered dose aerosol?

\section{Methods}

The study was of randomised, double blind, double dummy, parallel group design. 
CRITERIA FOR INCLUSION

Patients were eligible if they fulfilled the following criteria: (1) age from 18 to 70 years; (2) history of chronic asthma, with more than $15 \%$ improvement in forced expiratory volume in one second $\left(\mathrm{FEV}_{1}\right)$ after inhalation of a bronchodilator recorded in the previous 12 months; (3) requirement for more than $800 \mu \mathrm{g}$ inhaled corticosteroid daily; (4) baseline $\mathrm{FEV}_{1}$ above $20 \%$ of predicted normal value; (5) ability to use a metered dose aerosol efficiently. Patients were excluded if they were being treated with long term systemic corticosteroids or if they had received a short course of systemic corticosteroids in the preceding two weeks or more than six courses in the past 12 months.

\section{PROTOCOL}

Patients satisfying the criteria entered a four week run in period, during which they inhaled $750 \mu \mathrm{g}$ beclomethasone dipropionate twice daily from a metered dose aerosol without a spacer. They used a diary card to record: (1) sleep disturbance (yes/no); (2) morning and evening peak expiratory flow (PEF); (3) requirement for a bronchodilator inhaler (number of puffs); and (4) asthma symptoms (cough, wheeze, morning chest tightness, restriction of activities, and an overall symptom rating). Asthma symptoms were scored by the patient on a 0-9 scale, where $0=$ none and $9=$ very severe.

At the end of the run in period control of asthma was assessed from the diary card and tests of hypothalamo-pituitary-adrenal function (measurement of the $0900 \mathrm{~h}$ serum cortisol concentration and 24 hour urinary free cortisol excretion and the short tetracosactrin test) were performed. Adrenal axis function was judged normal if at least two of these test results were normal. ${ }^{3}$ Normal values were those established by the department of clinical chemistry in healthy subjects: $0900 \mathrm{~h}$ serum cortisol 190-550 nmol/l; urinary free cortisol $80-450 \mathrm{nmol} / 24 \mathrm{~h}$. A post-tetracosactrin cortisol concentration of $500 \mathrm{nmol} / 1$ and above was regarded as normal. The serum creatinine concentration was measured at the start of the study and was normal in all patients. Asthma was judged to be inadequately controlled if one or more of the following were present: (1) an overall symptom score of more than 4 on five or more of the previous seven days; (2) sleep disturbance because of asthma during two or more of the previous seven nights; (3) diurnal variation in PEF of $15 \%$ or more on five or more of the previous seven days; (4) 12 or more puffs of bronchodilator per 24 hours on five or more of the previous seven days.

Patients already taking $2 \mathrm{mg}$ beclomethasone dipropionate daily and otherwise eligible for study did not undergo a run in period and entered the randomised phase at $2 \mathrm{mg} /$ day, provided that hypothalamo-pituitary-adrenal function was normal. These patients and those with inadequately controlled asthma taking $1.5 \mathrm{mg}$ beclomethasone dipropionate who had normal hypothalamo-pituitaryadrenal function were randomised to receive increasing doses of beclomethasone dipropionate from a metered dose or without a large volume spacer (Volumatic). The daily dose was increased by $0.5 \mathrm{mg}$ at monthly intervals until hypothalamo-pituitary-adrenal suppression (results of two or more tests subnormal) developed or a dose of $5 \mathrm{mg}$ daily was reached. Aerosols delivering $250 \mu \mathrm{g}$ per actuation were used and therefore any patient reaching the highest dose was taking 40 puffs (20 active drug and 20 placebo) a day. The dosing frequency was twice daily initially but was increased to three times if a total dose of 14 puffs daily was reached. Patients were instructed to use the spacer first and to take two inhalations from the spacer after each actuation of the aerosol, each inhalation to be followed by a breath hold of five seconds. No instructions regarding mouth rinsing were given. Each month patients attended for clinic visits at $0900 \mathrm{~h}$ and collected urine for 24 hours before each visit. On these days they took the usual morning doses of inhaled steroid and placebo before attending. Immediately after venesection for the $0900 \mathrm{~h}$ cortisol estimation $0.25 \mathrm{mg}$ tetracosactrin was given intramuscularly and a further blood sample taken after 30 minutes. A throat swab was taken for culture for fungi and patients were questioned about any adverse events. Diary cards were checked and an assessment of compliance with the study medication was made from the remaining doses in returned canisters.

All patients gave written informed consent and the study was approved by the regional ethics committee.

\section{STATISTICAL ANALYSIS}

The study was analysed on the basis of intention to treat. The dose of beclomethasone dipropionate causing hypothalamo-pituitaryadrenal suppression was analysed by means of Wilcoxon rank sum tests and the $95 \%$ confidence interval for the difference between treatments was calculated. For all variables recorded on diary cards averages (means for PEF data, medians for symptom scores and use of relief bronchodilator) for the last available 14 days of each month of treatment were used in the analyses. Wilcoxon rank sum tests were used to compare spacer and non-spacer groups for asthma symptoms, proportion of nights disturbed by sleep, and use of relief bronchodilator. For PEF data $t$ tests were used. For all variables $p$ values below 0.05 were considered significant.

Before the study we expected a substantial difference in the dose of beclomethasone dipropionate causing hypothalamo-pituitaryadrenal suppression between the two groups and we thought that most of the patients treated without a spacer were likely to show hypothalamo-pituitary-adrenal suppression at a dose of $2.5 \mathrm{mg}$ beclomethasone dipropionate. Power calculations based on these assumptions suggested that 30 patients would 
need to be studied to give us an $80 \%$ chance of detecting a significant difference with $\mathrm{p}<0.05$.

\section{Results}

Fifty patients were recruited to the study, 11 of whom were taking $2 \mathrm{mg}$ beclomethasone dipropionate and one $2 \mathrm{mg}$ budesonide One patient whose asthma was well controlled by $1.5 \mathrm{mg}$ and whose adrenal axis function was normal was not included in the randomisation for treatment and was excluded from analysis. Of the 49 assigned a treatment code number, 24 were assigned to the group receiving the active drug via the spacer (S) and 25 to the non-spacer group (MDI). The demographic details of the two groups are shown in table 1 and their asthma medication at the start of the study in table 2. Four patients had previously required long term systemic corticosteroid treatment but this had been discontinued at least two years before the study.

Six of the 49 patients were found to have hypothalamo-pituitary-adrenal suppression and were therefore excluded from further study. Five were taking $1.5 \mathrm{mg}$ beclomethasone dipropionate daily and the sixth $2 \mathrm{mg}$. Five others were withdrawn because their asthma was well controlled during the run in period. Of the remaining 38, 20 were assigned to the $S$ group and 18 to the MDI group.

Of these 38 patients, 23 (14 S, $9 \mathrm{MDI}$ ) completed the study, one of whom (S) achieved the highest dose of $5 \mathrm{mg}$ daily without evidence of hypothalamo-pituitary-adrenal suppression. A second patient (MDI) also achieved the highest dose but proved to be not fully compliant. Another six patients ( $3 \mathrm{~S}$, $3 \mathrm{MDI}$ ) were withdrawn because of undercompliance with higher doses and three $(2 S)$ because of non-attendance. Three (2 MDI) withdrew from the study and two (MDI) were withdrawn because of persistently troublesome asthma symptoms.

\section{HYPOTHALAMO-PITUITARY-ADRENAL \\ FUNCTION TESTS}

The outcome in the 49 patients included in the intention to treat analysis is shown in the figure. The cumulative numbers of patients withdrawn at each visit, either because of hypothalamo-pituitary-adrenal suppression or for other reasons, are shown according to

Table 1 Details of the patients studied*

\begin{tabular}{lll}
\hline & Group & \\
\cline { 2 - 3 } & $\begin{array}{l}\text { Spacer }(S) \\
(n=24[20])\end{array}$ & $\begin{array}{l}\text { No spacer }(M D I) \\
(n=25[18])\end{array}$ \\
& $13: 11[12: 8]$ & $12: 13[7: 11]$ \\
Male:female & $46 \cdot 8(12 \cdot 6)[45 \cdot 2]$ & $43 \cdot 3(12 \cdot 6)[40 \cdot 6]$ \\
Mean age (y) (SD) & $1 \cdot 8(0 \cdot 2)[1 \cdot 83(0 \cdot 2)]$ & $1 \cdot 8(0 \cdot 2)[1 \cdot 77(0 \cdot 2)]$ \\
Body surface area (m², mean (SD)) & $9[7]$ & $9[6]$ \\
Smoker (n) & $19[15]$ & $19[16]$ \\
Atopic (n) & $17[15]$ & $19[16]$ \\
Duration of asthma $>10$ y $(n)$ & &
\end{tabular}

^Numbers in square brackets refer to patients entering the double blind phase of the study.
Table 2 Asthma medication at entry to study

\begin{tabular}{|c|c|c|}
\hline & \multicolumn{2}{|l|}{ Group } \\
\hline & $\begin{array}{l}\text { Spacer }(S) \\
(n=24)\end{array}$ & $\begin{array}{l}\text { No spacer }(M D I) \\
(n=25)\end{array}$ \\
\hline $\mathrm{BDP}(1-1.5 \mathrm{mg})$ & 15 & 14 \\
\hline $\mathrm{BDP}$ (2 mg/day) & 7 & 4 \\
\hline Budesonide $(1 \cdot 2-2 \mathrm{mg})$ & 2 & 7 \\
\hline Salbutamol & 16 & 23 \\
\hline Terbutaline & 8 & 2 \\
\hline Theophylline & 4 & 7 \\
\hline Controlled release salbutamol & 3 & 4 \\
\hline Cromoglycate & 2 & 1 \\
\hline $\begin{array}{l}\text { Nebulised salbutamol } \\
\text { and ipratropium }\end{array}$ & 0 & 1 \\
\hline
\end{tabular}

BDP_beclomethasone dipropionate.

treatment group. Analysis of the results of hypothalamo-pituitary-adrenal tests at each clinic visit suggested that there was no difference between the proportions of patients in each of the two groups who had hypothalamo-pituitary-adrenal suppression; on the basis of the last available results for each patient the proportions were $65 \%$ in $S v$ $66.7 \%$ in MDI (treatment difference $1.7 \%$, $95 \%$ confidence intervals $-30 \%$ to $33.4 \%$, p $=0.92$ ). The dose of beclomethasone causing hypothalamo-pituitary-adrenal suppression varied widely in both groups, ranging from 2.0 to $5.0 \mathrm{mg}$ in the spacer group and from 1.5 to $4.5 \mathrm{mg}$ in the MDI group. There was no difference in the median dose between the two groups $(3.25 \mathrm{mg}$ in $\mathrm{S} v 3.0 \mathrm{mg}$ in MDI; $\mathrm{p}$ $=0.99,95 \%$ confidence intervals for difference -1.0 to $1.0 \mathrm{mg}$ ).

DEVELOPMENT OF HYPOTHALAMO-PITUITARYADRENAL SUPPRESSION

Twenty nine of the 50 patients had evidence of hypothalamo-pituitary-adrenal suppression (results in at least two of the three screening tests subnormal), either at the initial screening or during the study. In $17(59 \%)$ of these patients all three tests yielded subnormal results. In the remaining 12 hypothalamopituitary-adrenal suppression was diagnosed on the basis of abnormal results for $0900 \mathrm{~h}$ cortisol and post-tetracosactrin cortisol in five, for $0900 \mathrm{~h}$ cortisol and urinary free cortisol in three, and for post-tetracosactrin cortisol and urinary free cortisol in four. No individual test was superior in detection of hypothalamo-pituitary-adrenal suppression. In most patients the pattern of suppression was reduced urinary cortisol excretion and $0900 \mathrm{~h}$ serum cortisol concentration with preservation of the response to tetracosactrin (an increment of at least $200 \mathrm{nmol} / \mathrm{l}$ ) but failure to achieve a value of $500 \mathrm{nmol} / 1$ or more because of a reduced basal value. The increment in cortisol was below $200 \mathrm{nmol} / \mathrm{l}$ in only nine $(31 \%)$ patients. Differences in the development of hypothalamo-pituitary-adrenal suppression were observed between patients: in some the serum and urinary cortisol declined progressively as the dose of beclomethasone was increased but in others a threshold effect was seen, the levels tending to remain well within normal ranges until a critical dose was reached. 


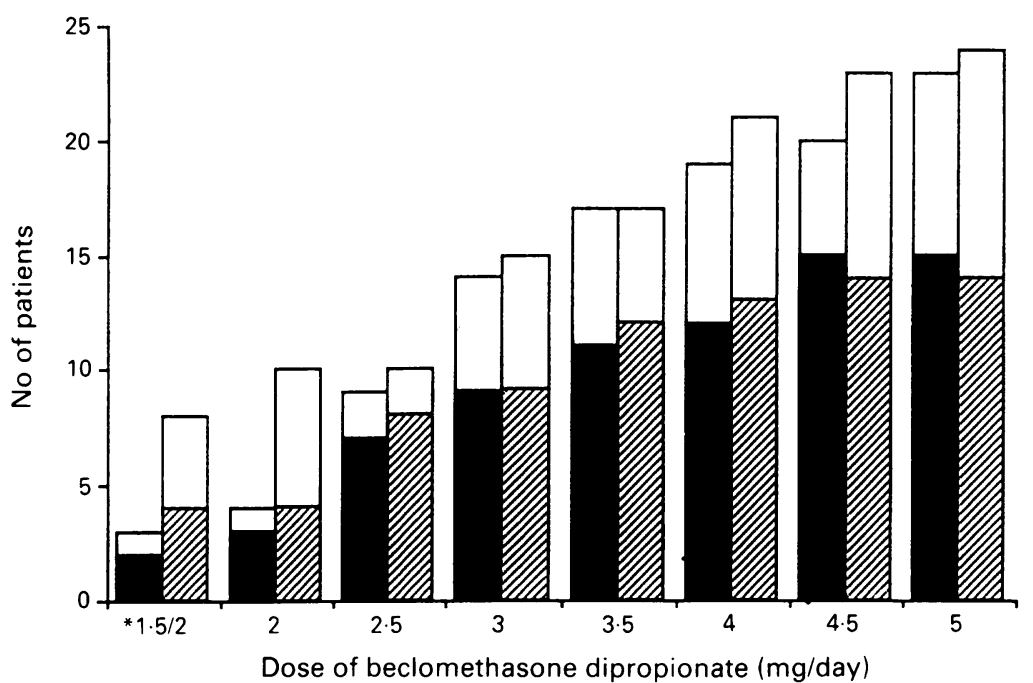

Cumulative number of patients withdrawn from study. $\square$ Group $S$ (initial $n=24$ ); $\nabla$ Group MDI $(n=25)$. The filled areas of the columns indicate patients withdrawn because of hypothalamo-pituitary-adrenal suppression and the open areas withdrawals for other reasons.

${ }^{\star}$ End of run in. at the next clinic visit. The remaining two patients (both $S$ ) required prednisolone and had suppressed hypothalamo-adrenal-pituitary function at the next visit; we could not therefore determine whether the higher dose of beclomethasone, treatment with prednisolone, or the combination was responsible for hypothalamo-pituitary-adrenal suppression.

\section{ADVERSE EFFECTS}

Adverse effects were mild and infrequent and none necessitated withdrawal from the study. The incidence of dysphonia and of throat discomfort was similar in the $S$ and MDI groups; these symptoms occurred in eight of the 49 patients but coexisted in only two. Five patients had clinical oropharyngeal candidiasis (4 MDI, $1 \mathrm{~S}$ ), of whom three (all MDI) had throat discomfort and one dysphonia. One patient (S) complained of transient light headedness after inhalation from active and placebo inhalers. Another complained of persistent cough after inhaling beclomethasone dipropionate via the spacer.

Systemic adverse effects of corticosteroids were observed in two patients (one in each treatment group). Both developed spontaneous bruising while taking beclomethasone dipropionate in doses ( 3.5 and $4.5 \mathrm{mg} /$ day) that caused subnormal hypothalamopituitary-adrenal function. Of the patients withdrawn from the study because of hypothalamo-pituitary-adrenal suppression, none developed symptoms of adrenal insufficiency with reduction in the dose of inhaled steroid.

\section{Discussion}

This study did not confirm that large volume spacer devices reduce the systemic effects of beclomethasone dipropionate inhaled in large doses, as measured by changes in hypothalamo-pituitary-adrenal axis function. The median dose of beclomethasone causing hypothalamo-pituitary-adrenal suppression was $3.25 \mathrm{mg}$ when the drug was administered through the spacer and $3.0 \mathrm{mg}$ when administered by the conventional metered dose aerosol. The wide $95 \%$ confidence intervals, however, indicate a high probability of type 2 error (the true difference between the two

Table 3 Asthma symptoms in patients inhaling $2 \mathrm{mg}$ beclomethasone dipropionate with and without a large volume spacer (median values except where otherwise specified)

\begin{tabular}{|c|c|c|c|c|}
\hline \multirow[b]{2}{*}{ Symptom } & \multicolumn{2}{|l|}{ Group } & \multirow[b]{2}{*}{$p$} & \multirow[b]{2}{*}{$\begin{array}{l}95 \% \text { CI for difference } \\
\text { between groups }\end{array}$} \\
\hline & $\begin{array}{l}\text { Spacer }(S) \\
(n=20)\end{array}$ & $\begin{array}{l}\text { No spacer }(M D I) \\
(n=15)\end{array}$ & & \\
\hline Cough & $2 \cdot 0$ & $1 \cdot 0$ & $0 \cdot 07$ & $(0.0$ to $2 \cdot 0)$ \\
\hline Wheeze & $2 \cdot 0$ & $2 \cdot 0$ & $0 \cdot 26$ & $(-0.5$ to 2.0$)$ \\
\hline Morning chest tightness & 1.5 & $2 \cdot 0$ & 0.9 & $(-1.0$ to 1.0$)$ \\
\hline Activity restriction & 1.5 & $2 \cdot 0$ & 0.41 & $(-1 \cdot 0$ to $2 \cdot 0)$ \\
\hline Overall symptom score & $2 \cdot 0$ & $2 \cdot 0$ & $0 \cdot 61$ & $(-1 \cdot 0$ to $2 \cdot 0)$ \\
\hline$\%$ nights with no sleep disturbance & $85 \cdot 2$ & $92 \cdot 9$ & $0 \cdot 8$ & $(-15 \cdot 0$ to $7 \cdot 0)$ \\
\hline Relief bronchodilator use (puffs/day) & $8 \cdot 0$ & $6 \cdot 0$ & 0.98 & $(-4 \cdot 0$ to $3 \cdot 0)$ \\
\hline \multicolumn{5}{|l|}{ PEF $(1 / \mathrm{min})^{\star}$} \\
\hline Morning & 340 & 341 & 0.97 & $(-73 \cdot 3$ to $71 \cdot 1)$ \\
\hline Evening & 361 & 362 & 0.98 & $(-75 \cdot 1$ to $73 \cdot 1)$ \\
\hline
\end{tabular}

*Mean values.

PEF-peak expiratory flow 
treatments, if one exists, could be as much as $1 \mathrm{mg}$ in favour of either mode of inhalation). The long duration of the study of up to nine months for an individual patient coupled with the requirement for patients to take an increasingly large number of puffs of trial medication resulted in a high withdrawal rate. In addition, there was a large variation between individuals in the extent to which the hypothalamo-pituitary-adrenal axis was suppressed by exogenous steroids. These two factors meant that insufficient patients achieved the study's endpoint to ensure adequate power.

For similar reasons and because asthma was generally well controlled while patients were taking $2 \mathrm{mg} /$ day, no conclusion can be drawn about whether high dose beclomethasone dipropionate is more effective if inhaled via a spacer. Mean morning and evening PEF values, however, were similar in the two groups while they were taking a dose of $2 \mathrm{mg}$ daily, suggesting that there is probably no difference in clinical efficacy at this dose. Consistent with this is the finding that bronchodilator use and symptom scores were very similar in the groups.

Few adverse effects were associated with high dose inhaled steroid treatment. The incidence of oropharyngeal candidiasis was similar to that previously reported with lower doses. ${ }^{89}$ Use of the spacer was associated with less throat irritation and clinical candidiasis but did not protect against dysphonia as has been reported..$^{10}$ Cough is probably related to aerosol propellants or surfactants and is reduced when steroid is inhaled as dry powder. ${ }^{11}$ Spontaneous bruising was rare $(4 \%)$ and occurred only at doses that caused hypthalamo-pituitary-adrenal suppression. Reports of bruising in patients taking high dose inhaled steroids ${ }^{12} 13$ have come from studies that did not include assessments of adrenal axis function, and it is not clear how this manifestation of the effects of systemic corticosteroid relates to suppression of endogenous steroid production.

In a previous study ${ }^{14}$ we found that 24 hour urinary excretion of less than $80 \mathrm{nmol}$ free cortisol and post-tetracosactrin cortisol concentrations below $500 \mathrm{nmol} / 1$ both correlated well with abnormal responses to insulin stress testing, widely regarded as the gold standard test for hypothalamo-pituitaryadrenal suppression. In contrast, the $0900 \mathrm{~h}$ serum cortisol concentration was a poor screening test. In the present study, however, at diagnosis of hypothalamo-pituitary-adrenal suppression the $0900 \mathrm{~h}$ cortisol was below $190 \mathrm{nmol} / \mathrm{l}$ in 25 of 29 patients, and this test was similar in sensitivity to the post-tetracosactrin cortisol concentration $(<500 \mathrm{nmol} / 1$ in $26 / 29)$ and urinary free cortisol $(<80 \mathrm{nmol} /$ $24 \mathrm{~h}$ in $24 / 29$ ). The rise in cortisol concentration in response to tetracosactrin proved, as in our previous study, to be a poor discriminator $(<200 \mathrm{nmol} / 1$ in only $9 / 29)$. The difference in the discriminant value of the $0900 \mathrm{~h}$ cortisol concentration between this study and the preceding one may be a reflection of the duration of hypothalamo-pituitary-adrenal suppression. The reduction in basal cortisol levels in blood and urine while the rise in cortisol concentration in the tetracosactrin test is preserved indicates that suppression caused by an increase in inhaled steroid is mainly occurring at the level of the hypothalamus or pituitary, or both. As the suppressive influence is maintained atrophy of the adrenal cortex occurs, and the ability of tetracosactrin to stimulate a prompt increase in cortisol secretion is lost. In the previous study ${ }^{14}$ the duration of adrenal axis suppression was unknown.

The current data sheet recommendations for the high dose beclomethasone dipropionate inhaler Becloforte state that the maximum daily dose should be $2 \mathrm{mg}$. This study confirms our earlier finding that $1.5 \mathrm{mg}$ beclomethasone dipropionate daily may be associated with subnormal adrenal axis function in a few patients. ${ }^{3}$ Hypothalamo-pituitary-adrenal function remained normal, however, in most patients until doses of more than $3 \mathrm{mg}$ a day were taken. The good control of asthma symptoms in most patients taking $2-2.5 \mathrm{mg}$ daily does not permit conclusions to be drawn about whether increasing the dose beyond this is associated with increased therapeutic efficacy. The upper limit of the dose-response curve for beclomethasone dipropionate (and for other inhaled corticosteroids) has not been established and, like susceptibility to hypothalamopituitary-adrenal suppression and other unwanted effects of steroids, is likely to vary widely between individual patients. Tests of hypothalamo-pituitary-adrenal function may be useful for predicting which patients will tolerate higher doses. In less severe exacerbations of asthma a temporary increase in inhaled steroid to $2 \cdot 5-3 \mathrm{mg}$ daily might be as effective as a short course of oral corticosteroids but associated with fewer long term adverse effects if frequent courses are required.

This study suggests that doses higher than the currently accepted maximum of $2 \mathrm{mg}$ beclomethasone dipropionate daily may be taken by many adults with asthma without causing subnormal hypothalamo-pituitaryadrenal function. Whether these higher doses control asthma symptoms more effectively remains to be established.

We thank Dr Gillian Blundell of the department of clinical chemistry, Western General Hospital, for performing the cortisol assays, Trudy Lowe of Allen and Hanburys Ltd for he help, and Margaret Keegan of Axess Ltd for performing the statistical analysis. PHB was supported by grants from Allen and Hanburys and Astra Pharmaceuticals Ltd.

1 Choo-Kang YFJ, Cooper EJ, Tribe AE, Grant IWB Beclomethasone dipropionate by inhalation in the treatment of airways obstruction. $\mathrm{Br} \mathcal{F}$ Dis Chest 1972;66: 101-6.

2 Smith MJ, Hodson ME. Effect of long term high dose beclomethasone dipropionate on adrenal function. Thorax 1983;38:676-81.

3 Brown PH, Blundell G, Greening AP, Crompton GK Hypothalamo-pituitary-adrenal axis suppression in asthmatics inhaling high dose corticosteroids. Respir Med 1991;85:501-10.

4 Prahl P, Jensen T. Decreased adreno-cortical suppression utilizing the Nebuhaler for inhalation of steroid aerosols. Clin Allergy 1987;17:393-8. 
5 Brown PH, Blundell G, Greening AP, Crompton GK. Do large volume spacer devices reduce the systemic effects of high dose inhaled corticosteroids? Thorax 1990;45:736-9.

6 Farrer M, Francis AJ, Pearce SJ. Morning serum cortisol concentration after $2 \mathrm{mg}$ inhaled beclomethasone dipropionate in normal subjects: effect of a $750 \mathrm{ml}$ spacing pionate in normal subjects: effect
device. Thorax 1990;45:740-2.

7 Selroos O, Halme M. Effect of a Volumatic spacer and mouth rinsing on systemic absorption of inhaled corticosteroids from a metered dose inhaler and dry powder inhaler. Thorax 1991;46:891-4.

8 Toogood JH, Baskerville J, Jennings B, Lefcoe $\mathrm{N}$, Johansson J-H. Use of spacers to facilitate inhaled corticosteroid treatment of asthma. Am Rev Respir Dis 1984; 129:723-9.

9 Milne LJR, Crompton GK. Beclomethasone dipropionate and oropharyngeal candidiasis. BMF 1974;iii:797-800.

10 Williamson IJ, Matusiewicz S, Brown P, Crompton GK,
Greening AP. Frequency of voice problems and cough in patients using aerosol steroid preparations [abstract]. Thorax 1991;46:769P.

11 Engel T, Heinig JH, Malling H-J, Scharling B, Nikander $\mathrm{K}$, Madsen F. Clinical comparison of inhaled budesonide delivered either by pressurised metered dose onide delivered either by pressurised meter

12 Capewell S, Reynolds S, Shuttleworth D, Edwards C, Finlay AY. Purpura and dermal thinning associated with high dose inhaled corticosteroids. BMF 1990;300: 1548-51.

13 Mak VHF, Melchor R, Spiro SG. Easy bruising as a side-effect of inhaled corticosteroids. Eur Respir $\mathcal{f}$ 1992;5:1068-74.

14 Brown PH, Blundell G, Greening AP, Crompton GK. Screening for hypothalamo-pituitary-adrenal suppression in asthmatics inhaling high dose corticosteroids. Respir Med 1991;85:511-6 